\title{
A JACOBIAN IDENTITY IN POSITIVE CHARACTERISTIC
}

\author{
JEFFREY LANG
}

\begin{abstract}
In this note, we present several new results on derivations in characteristic $p \neq 0$, together with a Jacobian identity that we recently discovered through a miscalculation. Our main identity states that, if $k$ is a field of characteristic $p$ and $f_{1}, \ldots, f_{n}$ belong to the polynomial ring $k\left[x_{1}, \ldots, x_{n}\right]$ and $J(f)$ equals the determinant of the $n \times n$ Jacobian matrix, $\left[\partial f_{i} / \partial x_{j}\right]$, then

$\sum_{i_{1}=1}^{p-1} \cdots \sum_{i_{n}=1}^{p-1} f_{1}^{i_{1}} \cdots f_{n}^{i_{n}} \nabla\left(f_{1}^{p-1-i_{1}} \cdots f_{n}^{p-1-i_{n}}\right)=(-1)^{n}(J(f))^{p-1}$,

where $\nabla=\partial^{n(p-1)} / \partial x_{1}^{p-1} \cdots \partial x_{n}^{p-1}$. We conclude with a brief discussion of nilpotent derivations in characteristic $p$ in connection with the degree less than $p$ version of the Jacobian conjecture.
\end{abstract}

Introduction. The genesis of this short paper was a computational error. If $k$ is a field of characteristic $p \neq 0$ and $f_{1}, f_{2}$ belong to the polynomial ring $A=k\left[x_{1}, x_{2}, x_{3}\right]$, we can define a derivation $D$ on $A$ by letting $D(h)$ be the determinant of the $3 \times 3$ Jacobian matrix $\partial\left(h, f_{1}, f_{2}\right) / \partial\left(x_{1}, x_{2}, x_{3}\right)$ for each $h \in A$. In [2, page 455], we showed that $D^{p}=\alpha_{D} D$, where $\alpha_{D}$ is given by the formula,

$$
\alpha_{D}=-\sum_{i=0}^{p-1} \sum_{j=0}^{p-1} f_{1}^{i} f_{2}^{j} \nabla\left(f_{1}^{p-1-i} f_{2}^{p-1-j}\right),
$$

where $\nabla=D_{1}^{p-1} D_{2}^{p-1} D_{3}^{p-1}$ with $D_{i}=\partial / \partial x_{i}$ for each $i$. For a specific application with $p=3$, we needed an explicit formula for $\alpha_{D}$ in terms of sums of products of mixed partial derivatives of the $f_{i}$. So we used the above formula but mistakenly replaced $\nabla$ by the operator $D_{1}^{p-1} D_{2}^{p-1}$.

2010 AMS Mathematics subject classification. Primary 13A35, 13N15.

Keywords and phrases. Jacobian, derivation, positive characteristic, nilpotent derivations, points at infinity.

Received by the editors on April 3, 2014.

DOI:10.1216/JCA-2015-7-3-393 Copyright (C)2015 Rocky Mountain Mathematics Consortium 
After a very long by hand computation we found to our surprise that

$$
\sum_{i=0}^{2} \sum_{j=0}^{2} f_{1}^{i} f_{2}^{j} D_{1}^{2} D_{2}^{2}\left(f_{1}^{2-i} f_{2}^{2-j}\right)=\left(\operatorname{det}\left(\partial\left(f_{1}, f_{2}\right) / \partial\left(x_{1}, x_{2}\right)\right)\right)^{2} .
$$

Based on this discovery, we conjectured that, if the characteristic of $k$ equals $p \neq 0$ and $\nabla=D_{1}^{p-1} D_{2}^{p-1}$, then we have for all $f_{1}, f_{2} \in k\left[x_{1}, x_{2}\right]$,

$$
\sum_{i=0}^{p-1} \sum_{j=0}^{p-1} f_{1}^{i} f_{2}^{j} \nabla\left(f_{1}^{p-1-i} f_{2}^{p-1-j}\right)=\left(\operatorname{det}\left(\partial\left(f_{1}, f_{2}\right) / \partial\left(x_{1}, x_{2}\right)\right)\right)^{p-1} \text {. }
$$

Attempts to verify this combinatorially proved very difficult, but our discovery of a general identity (Proposition 1.3) involving derivations in characteristic $p$ made things considerably simpler, leading to the proof of the above identity and its generalization to higher dimension (Theorem 1.7). While we have not yet found applications for it, we hope that it will be useful in the study of logarithmic derivatives of Jacobian derivations in characteristic $p$ and that it may shed further light on the Jacobian condition in positive characteristic.

The remainder of the first section has to do with the fact that the rings $A=k\left[x_{1}, \ldots, x_{n}\right]$ and $B=k\left[x_{1}^{p}, \ldots, x_{n}^{p}, f_{1}, \ldots, f_{n}\right]$ have the same quotient fields when the determinant of the $n \times n$ Jacobian matrix, $\left[D_{i} f_{j}\right]$, is nonzero. Hence, if $J(f)=\operatorname{det}\left[D_{i} f_{j}\right]$ and $J(f) \neq 0$, then the two rings will be the same after inversion of some element in $B$. In Proposition 1.12, we show that $(J(f))^{p}$ fulfills this role; that is, if $J(f) \neq 0$, then

$$
B\left[\frac{1}{(J(f))^{p}}\right]=A\left[\frac{1}{J(f)}\right] .
$$

In the second section, we discuss attempting the two variable Jacobian conjecture via a reduction to characteristic $p$. The two variable Jacobian conjecture is not true in positive characteristic $p$, but it is not known if it is true for polynomials of degree less than $p$. If it is true in this case, it would imply the two variable Jacobian conjecture in characteristic 0 [3, page 273]. Yet, if the degree less than $p$ Jacobian conjecture is indeed true in characteristic $p$ and, if a reduction to this scenario is likely to produce results, then it is our view that one should be able to recover, without enormous difficulty, in characteristic $p$ for Jacobian pairs of degree less than $p$ already established 
algebraic properties of Jacobian pairs in characteristic 0. Along these lines, we consider the number of points at infinity of polynomials in characteristic $p$ of degree less than $p$ that belong to Jacobian pairs, for we know from Abyankar [1, page 139] that, in characteristic 0, Jacobian pairs have at most two points at infinity. At first glance, the natural category of polynomials of degree less than $p$ to consider for recovering Abhyankar's theorem are those that define nilpotent Jacobian derivations, since it includes those that belong to Jacobian pairs and since they possess the essential property (Lemma 2.1) that in characteristic 0 forces their highest degree forms to have at most two irreducible factors (Lemma 2.2). Nevertheless, using methods from the first section, we show that such a polynomial can have as many points at infinity as its degree (Proposition 2.8), from which we surmise that tackling the Jacobian problem in characteristic $p$ for the degree less than $p$ case is probably going to be at least as hard as solving the characteristic 0 case (Conclusion 2.10).

1. A Jacobian identity. In this section, we let $k$ be a field of characteristic $p \neq 0, A=k\left[x_{1}, \ldots, x_{n}\right]$ a polynomial ring in $n$ indeterminates over $k$, and $L=k\left(x_{1}, \ldots, x_{n}\right)$ the quotient field of $A$. For each $i=1,2, \ldots, n$, let $D_{i}=\partial / \partial x_{i}$, and let $\nabla$ be the differential operator on $L$ given by $\nabla=D_{1}^{p-1} \cdots D_{n}^{p-1}$. Given $f_{1}, \ldots, f_{n} \in A$, let $J\left(f_{1}, \ldots, f_{n}\right)$ be the determinant of the $n \times n$ Jacobian matrix,

$$
\partial\left(f_{1}, \ldots, f_{n}\right) / \partial\left(x_{1}, \ldots, x_{n}\right)=\left[\begin{array}{ccc}
D_{1}\left(f_{1}\right) & \cdots & D_{1}\left(f_{n}\right) \\
\vdots & & \vdots \\
D_{n}\left(f_{1}\right) & \cdots & D_{n}\left(f_{n}\right)
\end{array}\right] .
$$

When $B$ is a commutative ring with unity, we let $B^{*}$ denote the multiplicative groups of units in $B$.

Definition 1.1. For each pair of nonnegative integers $n, k$, let $B_{n, k}$ denote the Bell polynomial in $n-k+1$ variables, which is given by

$$
B_{n, k}=\sum \frac{n !}{j_{1} ! j_{2} ! \cdots j_{n-k+1} !}\left(\frac{x_{1}}{1 !}\right)^{j_{1}}\left(\frac{x_{2}}{2 !}\right)^{j_{2}} \cdots\left(\frac{x_{n-k+1}}{(n-k+1) !}\right)^{j_{n-k+1}}
$$

where the sum is over all sequences $j_{1}, j_{2}, j_{3} \ldots, j_{n-k+1}$ of nonnegative integers such that $\sum j_{i}=k$ and $\sum i j_{i}=n$. 
Lemma 1.2. Let $R$ be a ring of characteristic $p \neq 0$, and let $D$ be a derivation on $R$. Then, for each $a \in R$ and positive integer $r$, we have

$$
D^{p-1}\left(a^{r}\right)=\sum_{k=0}^{p-1}\left(\begin{array}{l}
r \\
k
\end{array}\right) k ! B_{p-1, k}\left(D a, D^{2} a, \ldots, D^{p-1} a\right) \cdot a^{r-k} .
$$

Proof. Let $f(x) \in R[x]$ be given by $f(x)=x^{r}$. By Faà di Bruno's formula,

$$
D^{p-1}\left(a^{r}\right)=\sum_{k=0}^{p-1} f^{[k]}(a) B_{p-1, k}\left(D a, D^{2} a, \ldots, D^{p-1} a\right),
$$

which yields the result, since the $k$ th derivative of $f(x)$ is given by $f^{[k]}(x)=\left(\begin{array}{l}r \\ k\end{array}\right) k ! x^{r-k}$.

The Jacobian identity (Theorem 1.7) below is essentially a special case of this next proposition, which is employed several times in this article.

Proposition 1.3. Let $R$ be a ring of characteristic $p \neq 0$, and let $D: R \rightarrow R$ be a derivation on $R$. Then, for each $a \in R$, we have

$$
\sum_{r=0}^{p-1} a^{p-1-r} D^{p-1}\left(a^{r}\right)=-(D a)^{p-1} .
$$

Proof. Let $a \in R$. It follows from Lemma 1.2 that

$$
\begin{aligned}
& \sum_{r=0}^{p-1} a^{p-1-r} D^{p-1}\left(a^{r}\right) \\
& \quad=\sum_{r=0}^{p-1} \sum_{k=0}^{p-1}\left(\begin{array}{l}
r \\
k
\end{array}\right) k ! a^{p-1-k} B_{p-1, k}\left(D a, D^{2} a, \ldots, D^{p-1} a\right) \\
& \quad=\sum_{k=0}^{p-1}\left(\begin{array}{c}
p \\
k+1
\end{array}\right) k ! a^{p-1-k} B_{p-1, k}\left(D a, D^{2} a, \ldots, D^{p-1} a\right) \\
& \quad=(p-1) ! B_{p-1, p-1}\left(D a, D^{2} a, \ldots, D^{p-1} a\right) .
\end{aligned}
$$

Since

$$
B_{p-1, p-1}=\frac{(p-1) !}{(p-1) !} x_{1}^{p-1}
$$


we obtain

$$
\sum_{r=0}^{p-1} a^{p-1-r} D^{p-1}\left(a^{r}\right)=-(D a)^{p-1}
$$

Corollary 1.4. Let $R$ be a ring of characteristic $p \neq 0$, and let $D: R \rightarrow R$ be a derivation on $R$. Assume that either $p=2$ or $R$ has no nonzero nilpotents. If $D \neq 0$, then $D^{p-1} \neq 0$.

Proof. If $D^{p-1}=0$ on $R$, then, for each $a \in R, D a=0$, by Proposition 1.3.

Proposition 1.5. Let $f_{1}, \ldots, f_{n-1} \in L$ and $D$ be the derivation on $L$ defined by $D(t)=J\left(t, f_{1}, \ldots, f_{n-1}\right)$ for all $t \in L$. If $D \neq 0$, then there exists $\alpha_{D} \in D^{-1}(0)$ such that $D^{p}=\alpha_{D} D$ and, for all $t \in L$,

$$
D^{p-1}(t)-\alpha_{D} t=(-1)^{n-1} \sum_{\substack{i_{j}=0 \\ 0 \leq j \leq n-1}}^{p-1} f_{1}^{i_{1}} \cdots f_{n-1}^{i_{n-1}} \nabla\left(f_{1}^{p-1-i_{1}} \cdots f_{n-1}^{p-1-i_{n-1}} t\right) .
$$

Proof. In [2, Proposition 2.1], we proved Proposition 1.5 for the case where $f_{1}, \ldots, f_{n-1} \in A$. Specifically, we showed that if $f_{1}, \ldots, f_{n-1} \in$ $A$ and $D$ is the derivation on $L$ defined as above with $D \neq 0$, then there exists $\alpha_{D} \in D^{-1}(0) \cap A$ such that $D^{p}=\alpha_{D} D$ and, for all $t \in L$,

$$
D^{p-1}(t)-\alpha_{D} t=(-1)^{n-1} \sum_{\substack{i_{j}=0 \\ 0 \leq j \leq n-1}}^{p-1} f_{1}^{i_{1}} \cdots f_{n-1}^{i_{n-1}} \nabla\left(f_{1}^{p-1-i_{1}} \cdots f_{n-1}^{p-1-i_{n-1}} t\right) \text {. }
$$

The version presented in this proposition follows immediately from this and the fact that, for each $f \in L$, there exists $g, h \in A$ such that $f=g / h^{p}$.

Lemma 1.6. Let $f_{1}, \ldots, f_{n-1} \in L$ and $D$ be the derivation on $L$ defined by $D(t)=J\left(t, f_{1}, \ldots, f_{n-1}\right)$ for all $t \in L$. Then the following are equivalent:

(i) $D \neq 0$ on $L$.

(ii) $\left[L^{(p)}\left(f_{1}, f_{2}, \ldots, f_{n-1}\right): L^{(p)}\right]=p^{n-1}$, where $L^{(p)}=k\left(x_{1}^{p}, \ldots, x_{n}^{p}\right)$.

(iii) The Jacobian matrix $\partial\left(f_{1}, \ldots, f_{n-1}\right) / \partial\left(x_{1}, \ldots, x_{i-1}, x_{i+1}, \ldots, x_{n}\right)$ has nonzero determinant for some $i=1,2, \ldots, n$. 
Proof. The equivalence of the first and third statement follows from the fact that, for each $i, D\left(x_{i}\right)$ equals \pm the described determinant. The equivalence of the first and second statement follows from the fact that, for $f_{1}, \ldots, f_{n} \in L, J\left(f_{1}, \ldots, f_{n}\right) \neq 0$ if and only if no $f_{i}$ belongs to $L^{(p)}\left(f_{1}, \ldots, f_{i-1}, f_{i+1}, \ldots, f_{n}\right)$, and the latter is clearly equivalent to the statement that $L^{(p)}\left(f_{1}, \ldots, f_{n}\right)=L$.

Theorem 1.7. Let $f_{1}, \ldots, f_{n} \in L=k\left(x_{1}, \ldots, x_{n}\right)$. Then,

$$
\begin{aligned}
\sum_{i_{1}=1}^{p-1} \cdots \sum_{i_{n}=1}^{p-1} f_{1}^{i_{1}} \cdots f_{n}^{i_{n}} \nabla\left(f_{1}^{p-1-i_{1}} \cdots f_{n}^{p-1-i_{n}}\right) & \\
& =(-1)^{n}\left(J\left(f_{1}, \ldots, f_{n}\right)\right)^{p-1} .
\end{aligned}
$$

Proof. For notational convenience, for each $f_{1}, \ldots, f_{n} \in L$, let

$$
T\left(f_{1}, \ldots, f_{n}\right)=\sum_{i_{1}=1}^{p-1} \cdots \sum_{i_{n}=1}^{p-1} f_{1}^{i_{1}} \cdots f_{n}^{i_{n}} \nabla\left(f_{1}^{p-1-i_{1}} \cdots f_{n}^{p-1-i_{n}}\right) .
$$

We consider two cases.

Case 1. Assume $J\left(f_{1}, \ldots, f_{n}\right) \neq 0$. Then $\left[L^{(p)}\left(f_{2}, \ldots, f_{n}\right): L^{(p)}\right]=$ $p^{n-1}$. Let $D$ be the derivation on $L$ defined by $D(t)=J\left(t, f_{2}, \ldots, f_{n}\right)$ for all $t \in L$. Then $D \neq 0$. Hence, for each $r=0,1, \ldots, p-1$, we have by Proposition 1.5,

$$
\begin{aligned}
& D^{p-1}\left(f_{1}^{p-1-r}\right)-\alpha_{D} f_{1}^{p-1-r} \\
& \quad=(-1)^{n-1} \sum_{i_{j}=0}^{p-1} f_{2}^{i_{2}} \cdots f_{n}^{i_{n}} \nabla\left(f_{2}^{p-1-i_{2}} \cdots f_{n}^{p-1-i_{n}} f_{1}^{p-1-r}\right) .
\end{aligned}
$$

After multiplying both sides by $f_{1}^{r}$, we obtain

$$
\begin{aligned}
f_{1}^{r} D^{p-1} & \left(f_{1}^{p-1-r}\right)-\alpha_{D} f_{1}^{p-1} \\
= & (-1)^{n-1} \sum_{i_{j}=0}^{p-1} f_{1}^{r} f_{2}^{i_{2}} \cdots f_{n}^{i_{n}} \nabla\left(f_{1}^{p-1-r} f_{2}^{p-1-i_{2}} \cdots f_{n}^{p-1-i_{n}}\right) .
\end{aligned}
$$


If we sum both sides of this last equality for $r=0,1, \ldots, p-1$ and apply Proposition 1.3, we obtain the desired formula.

Case 2. The general case. If some $f_{i} \in k$, then $J\left(f_{1}, \ldots, f_{n}\right)=0$. We therefore need to show that $T\left(f_{1}, \ldots, f_{n}\right)=0$ in this case. Without loss of generality, we may assume $f_{n} \in k$. Then, since $D_{i}\left(f_{n}\right)=0$ for each $i$, the summation on the left of the proposed identity becomes

$$
\sum_{i_{n}=0}^{p-1} \sum_{\substack{i_{j}=0 \\ 0 \leq j \leq n-1}}^{p-1} f_{1}^{i_{1}} \cdots f_{n-1}^{i_{n-1}} f_{n}^{p-1} \nabla\left(f_{1}^{p-1-i_{1}} \cdots f_{n-1}^{p-1-i_{n-1}}\right),
$$

which equals 0 , since the inner sum in this double summation is independent of $i_{n}$.

Therefore, we can assume that, for each $i=1,2, \ldots, n, f_{i}$ has positive degree $n_{i}$. For each $n$-tuple of nonnegative integers, $w=$ $\left(e_{1}, \ldots, e_{n}\right)$, let $x^{w}=x_{1}^{e_{1}} \cdots x_{n}^{e_{n}}$. For each $i=1,2, \ldots n$, let $W_{i}$ be the set of all $n$-tuples of nonnegative integers such that the sum of their entries is at most $n_{i}$. Let $\mathbf{T}=\left\{T_{i, w}: 1 \leq i \leq n, w \in W_{i}\right\}$ be a set of indeterminates over $k$. Let $K=k(\mathbf{T})$ be the field extension of $k$ generated by the elements of $\mathbf{T}$. For each $i=1,2, \ldots, n$, let $F_{i}=\sum_{w \in W_{i}} T_{i, w} x^{w}$. Then $J\left(F_{1}, \ldots, F_{n}\right) \neq 0$, since this is so for a generic choice of specializations of the $F_{i}$, and hence, $T\left(F_{1}, \ldots, F_{n}\right)=$ $(-1)^{n}\left(J\left(F_{1}, \ldots, F_{n}\right)\right)^{p-1}$ by the above case. Therefore, the same is true for any specialization of the $F_{1}, \ldots, F_{n}$, in particular, for the specialization that produces $f_{1}, \ldots, f_{n}$.

Throughout the rest of this section, $K$ will be a field of characteristic $p \neq 0$ and $D: K \rightarrow K$ a derivation. Let $K^{\prime}=D^{-1}(0)$, and let $\mathbb{F}_{p}$ denote the prime subfield of $K$.

The following is from Pierre Samuel's Tata notes.

Proposition 1.8. If $\left[K: K^{\prime}\right]=p$, then there exists $a \in K^{\prime}$ such that $D^{p}=a D[4$, page 63$]$.

The next lemma is a more general version of one [4, Lemma 2.3] that was used to prove some equivalences to the Jacobian condition in positive characteristic in terms of the $\nabla$ operator. 
Lemma 1.9. If $\left[K: K^{\prime}\right]=p$ and $f \in K$, then $D(f) \in \mathbb{F}_{p}^{*}$ if and only if, for each $h \in K$,

$$
h+\sum_{i=0}^{p-1} f^{p-1-i} D^{p-1}\left(f^{i} h\right)=0 .
$$

Proof. Assume that $D(f) \in \mathbb{F}_{p}^{*}$, and let $b=D(f)$.

Note that $D(1)=D\left(1^{2}\right)=2 D(1)$. Hence, $D(1)=0$ and, by the additivity of $D$, we have $D(b)=0$. Thus, for each pair of nonnegative integers $r$ and $s$, we have $D^{r}\left(f^{s}\right)=r !\left(\begin{array}{l}s \\ r\end{array}\right) f^{s-r} b^{r}$.

Let $h \in K$, and let $\beta=\sum_{i=0}^{p-1} f^{p-1-i} D^{p-1}\left(f^{i} h\right)$. Then

$$
\begin{aligned}
\beta & =\sum_{i=0}^{p-1} f^{p-1-i} \sum_{j=0}^{p-1}\left(\begin{array}{c}
p-1 \\
j
\end{array}\right) D^{j}\left(f^{i}\right) D^{p-1-j}(h) \\
& =\sum_{i=0}^{p-1} \sum_{j=0}^{p-1}(-1)^{j}\left(\begin{array}{l}
i \\
j
\end{array}\right)(j !) b^{j} f^{p-1-j} D^{p-1-j}(h) \\
& =\sum_{j=0}^{p-1} \sum_{i=0}^{p-1}(-1)^{j}\left(\begin{array}{l}
i \\
j
\end{array}\right)(j !) b^{j} f^{p-1-j} D^{p-1-j}(h) \\
& =\sum_{j=0}^{p-1}(-1)^{j}(j !) b^{j} f^{p-1-j} D^{p-1-j}(h) \sum_{i=0}^{p-1}\left(\begin{array}{c}
i \\
j
\end{array}\right) \\
& =\sum_{j=0}^{p-1}(-1)^{j}(j !) b^{j} f^{p-1-j} D^{p-1-j}(h)\left(\begin{array}{c}
p \\
j+1
\end{array}\right) .
\end{aligned}
$$

Since $\left(\begin{array}{c}p \\ j+1\end{array}\right)=0$ unless $j=p-1, \beta=(-1)^{p-1}(p-1) ! b^{p-1} f^{0} D^{0}(h)=$ $-h$.

For the converse, assume that, for all $h \in K$,

$$
h+\sum_{i=0}^{p-1} f^{p-1-i} D^{p-1}\left(f^{i} h\right)=0 .
$$


Then, in particular,

$$
\begin{aligned}
f & =-\sum_{i=0}^{p-1} f^{p-1-i} D^{p-1}\left(f^{i+1}\right) \\
& =-\sum_{i=0}^{p-2} f^{p-1-i} D^{p-1}\left(f^{i+1}\right) \\
& =-f \sum_{i=0}^{p-2} f^{p-2-i} D^{p-1}\left(f^{i+1}\right)=f(D f)^{p-1},
\end{aligned}
$$

where we obtain the last equality by Proposition 1.3. Hence, $(D f)^{p-1}=$ 1, which implies $D f \in \mathbb{F}_{p}^{*}$.

The following appears in [3]. We include a proof here for the sake of completeness.

Lemma 1.10. [3, page 276]. Let $R$ be a subring of $K$ such that $D(R) \subset R$, and let $R^{\prime}=R \cap K^{\prime}$. If $f \in R$ is such that $D f \in R^{*}$, then $R=R^{\prime}[f]$.

Proof. Let $\triangle=(D f)^{-1} D$. By Proposition 1.8, there exists $a \in K^{\prime}$ such that $\triangle^{p}=a \triangle$. Since $\triangle f=1, a=0$. Thus, $\triangle^{p-1}(R) \subset R^{\prime}$. It then follows by Lemma 1.9 that, for each $h \in R$,

$$
h=-\sum_{i=0}^{p-1} f^{p-1-i} \triangle^{p-1}\left(f^{i} h\right) \in R^{\prime}[f] .
$$

Proposition 1.11. Assume $R$ is as in Lemma 1.10 and $f \in R$. If $D f \neq 0$, then

$$
R^{\prime}\left[f, \frac{1}{(D f)^{p}}\right]=R\left[\frac{1}{D f}\right]
$$

Proof. Define a derivation $\triangle$ on $K$ by $\triangle=1 / D f \cdot D$. Then

$$
\triangle\left(R\left[\frac{1}{D f}\right]\right) \subset R\left[\frac{1}{D f}\right],
$$


and we also have

$$
R^{\prime}\left[\frac{1}{(D f)^{p}}\right] \subset K^{\prime} \cap R\left[\frac{1}{D f}\right] .
$$

To show the reverse containment with regard to the latter, let $b \in$ $K^{\prime} \cap R[1 / D f]$. Then there exists $a \in R$ and a nonnegative integer $n$ such that $b=a /(D f)^{n}$. Multiplying the numerator and denominator by an appropriate power of $D f$, we may assume $n$ is a multiple of p. Then $\triangle b=0$ implies $\triangle a=0$, i.e., $b \in R^{\prime}\left[1 /(D f)^{p}\right]$. Hence, $R^{\prime}\left[1 /(D f)^{p}\right]=K^{\prime} \cap R[1 / D f]$ and, since $\triangle f=1$, we obtain

$$
R^{\prime}\left[f, \frac{1}{(D f)^{p}}\right]=R\left[\frac{1}{D f}\right]
$$

by Lemma 1.10 .

Proposition 1.12. Let $A=k\left[x_{1}, \ldots, x_{n}\right]$ be a polynomial ring in $n$ variables and $f_{1}, \ldots, f_{n} \in A$. Let $B=k\left[x_{1}^{p}, \ldots, x_{n}^{p}, f_{1}, \ldots, f_{n}\right]$. If $J\left(f_{1}, \ldots, f_{n}\right) \neq 0$, then

$$
A\left[\frac{1}{J\left(f_{1}, \ldots, f_{n}\right)}\right]=B\left[\frac{1}{J\left(f_{1}, \ldots, f_{n}\right)^{p}}\right]=B\left[\frac{1}{J\left(f_{1}, \ldots, f_{n}\right)}\right] .
$$

Proof. Let $A^{\prime}=k\left[x_{1}^{p}, \ldots, x_{n}^{p}, f_{2}, \ldots, f_{n}\right]$, and let $L$ and $L^{\prime}$ denote the quotient fields of $A$ and $A^{\prime}$, respectively. Let $D$ be the derivation on $L$ defined by $D(h)=J\left(h, f_{2}, \ldots, f_{n}\right)$ for all $h \in L$. Since $D\left(f_{1}\right)=$ $J\left(f_{1}, \ldots, f_{n}\right) \neq 0$, we have $L^{\prime}=D^{-1}(0)$ and $\left[L: L^{\prime}\right]=p$. By a similar argument as that used in the proof of Proposition 1.11, we have

$$
A^{\prime}\left[\frac{1}{\left(D f_{1}\right)^{p}}\right]=L^{\prime} \cap A\left[\frac{1}{D f_{1}}\right] \text {. }
$$

We then have

$$
B\left[\frac{1}{\left(J\left(f_{1}, \ldots, f_{n}\right)\right)^{p}}\right]=A^{\prime}\left[f_{1}, \frac{1}{\left(D f_{1}\right)^{p}}\right]=A\left[\frac{1}{D f_{1}}\right]=A\left[\frac{1}{J\left(f_{1}, \ldots, f_{n}\right)}\right],
$$

with the middle equality occurring as a result of Proposition 1.11. Since $\left(J\left(f_{1}, \ldots, f_{n}\right)\right)^{p-1} \in B$ by Theorem 1.7 , we have

$$
B\left[\frac{1}{J\left(f_{1}, \ldots, f_{n}\right)}\right]=B\left[\frac{1}{\left(J\left(f_{1}, \ldots, f_{n}\right)\right)^{p}}\right] .
$$


2. Points at infinity of nilpotent Jacobian derivations. In this section, we let $k$ be a field and $k[x, y]$ a polynomial ring in two variables. A pair of polynomials $f, g \in k[x, y]$ will be referred to as a Jacobian pair if $J(f, g)=f_{x} g_{y}-f_{y} g_{x} \in k^{*}$, in which case we also say that $f$ (as well as $g$ ) is part of a Jacobian pair. For each $f \in k[x, y]$, we let $D_{f}$ denote the derivation on $k(x, y)$ defined by $D_{f}(h)=J(f, h)$ for each $h \in k(x, y)$. For $f \in k[x, y]$ with $f \neq 0$, we let $f^{+}$denote the nonzero homogeneous form of $f$ of highest degree, and we say that $f$ has $r$ points at infinity if $f^{+}$is a product of $r$ mutually coprime factors in $\bar{k}[x, y]$, where $\bar{k}$ is an algebraic closure of $k$. A pair of polynomials $f, g \in k[x, y]$ will be referred to as an automorphic pair if $k[f, g]=k[x, y]$. The two variable Jacobian conjecture states that if the characteristic of $k$ equals 0 , then every Jacobian pair in $k[x, y]$ is an automorphic pair.

It is not hard in positive characteristic to produce examples of Jacobian pairs that are not automorphic pairs. For example, if $k$ is a field of characteristic $p \neq 0$ and $u \in k[x, y]$ has positive degree, then $J\left(x, y+u^{p} x\right)=1$, but $k[x, y] \neq k\left[x, y+u^{p} x\right]$, since $y+u^{p} x$ will have more than one point at infinity [1, page 95]. Also, in characteristic $p \geq 3, f=x^{(p-1) / 2} y^{(p+1) / 2}+y, g=(x+y) x^{(p-1) / 2} y^{(p-1) / 2}+y-x$ is an example of a Jacobian non-automorphic pair of degree $p$, since $f$ has two and $g$ has three points at infinity. However, in characteristic $p \neq 0$, there are no known Jacobian pairs with degrees of the two polynomials both less than $p$ that are not also automorphic pairs. Yet, if none such exist, or if the polynomials of such pairs always have only one point at infinity, or have triangular Newton polygons, then the two variable Jacobian conjecture is true in characteristic 0 [3, page 273]. For this reason and because we have a fairly good understanding of Jacobian $n$-tuples in positive characteristic along with some analytical tools for determining them [3, pages 274-275], the study in characteristic $p \neq 0$ of polynomials in two variables of degree less than $p$ that occur as parts of Jacobian pairs would seem to hold promise. But, after numerous unfruitful attempts over the years along these lines at the two variable Jacobian conjecture, it occurred to us to test the overall feasibility of this approach by attempting to recover in characteristic $p$ for polynomials of degree less than $p$, an established fact concerning parts of Jacobian pairs in characteristic 0 , specifically, Abhyankar's theorem on their number of points at infinity [1, pages 138-139]. For, if such a result in characteristic 0 cannot be obtained in 
characteristic $p$ for polynomials of degree less than $p$, then we should probably be doubtful of there being an advantage to a degree less than $p$ approach to proving the two variable Jacobian conjecture.

Abhyankar's proof that a polynomial $f \in k[x, y]$ that is part of a Jacobian pair has at most two points at infinity, depends heavily on the assumption that the characteristic of $k$ is 0 and essentially reduces to two steps [1, pages 120-136], which we list here as lemmas.

Lemma 2.1. Let $k$ be a field of characteristic 0 , and let $f \in k[x, y]$. If there exists $g \in k[x, y]$ such that $J(f, g) \neq 0$ and $J\left(f^{+},(J(f, g))^{+}\right)=0$, then there exists a homogeneous $H \in k[x, y]$ such that $D_{f^{+}}(H)=$ $J\left(f^{+}, H\right) \neq 0$ and $D_{f^{+}}^{2}(H)=J\left(f^{+}, J\left(f^{+}, H\right)\right)=0$.

Lemma 2.2. Let $k$ be a field of characteristic 0 , and let $F \in k[x, y]$ be homogeneous. If there exists a homogeneous $H \in k[x, y]$ such that $D_{F}(H)=J(F, H) \neq 0$ and $D_{F}^{2}(H)=J(F, J(F, H))=0$, then $F$ has at most two mutually coprime factors.

Certainly, if $f, g \in k[x, y]$ and $J(f, g) \in k^{*}$, then $J\left(f^{+},(J(f, g))^{+}\right)=$ 0 , so that by Lemmas 2.1 and $2.2, f^{+}$will have at most two mutually coprime factors, i.e., $f$ will have at most two points at infinity.

We will see below that, if the characteristic of $k$ is $p \neq 0$ and $f \in k[x, y]$, then the existence of an $h \in k[x, y]$ such that $D_{f}(h) \neq 0$ and $D_{f}^{2}(h)=0$ is equivalent to the condition that $D_{f}$ is nonzero and nilpotent on $k(x, y)$ (Corollary 2.4). Hence, in particular, if $f$ is part of a Jacobian pair, then $D_{f}$ is nonzero and nilpotent on $k(x, y)$. We will also show (Corollary 2.7) that, if $f^{+} \notin k\left[x^{p}, y^{p}\right]$ and $D_{f}$ is nilpotent on $k(x, y)$, then there exists a homogeneous $H \in k[x, y]$ such that $D_{f^{+}}(H)=J\left(f^{+}, H\right) \neq 0$ and $D_{f^{+}}^{2}(H)=J\left(f^{+}, J\left(f^{+}, H\right)\right)=0$. Therefore, if $\operatorname{deg}(f)<p$ and $f$ is part of a Jacobian pair, then the conclusion of Lemma 2.1 holds for $f$. Since obtaining this conclusion in characteristic 0 is the trickier step in Abhyankar's proof, this seems like cause for optimism.

Nevertheless, the question then becomes, is Lemma 2.2 true for homogeneous $F \in k[x, y]$ of degree less than $p$ ? In Proposition 2.8, we use a characterization of nilpotent derivations in terms of the differential operator $\nabla=\partial^{2(p-1)} / \partial x^{p-1} \partial y^{p-1}$ (Proposition 2.6) to 
show that there are many homogeneous $F$ of degree less than $p$ with many more than two mutually coprime factors for which there exists homogeneous $H \in k[x, y]$ with $D_{F}(H)=J(F, H) \neq 0$ and $D_{F}^{2}(H)=$ $J(F,(J(F, H)))=0$. Thus, even when $\operatorname{deg}(F)<p$, Lemma 2.2 above is not true. This suggests to us that proving that polynomials of degree less than $p$ that are parts of Jacobian pairs have at most two points at infinity, provided this is true, will require a completely novel approach and should be quite challenging (Conclusion 2.10).

For the next two propositions, we assume $K$ is a field of characteristic $p \neq 0, D: K \rightarrow K$ is a derivation, $K^{\prime}=D^{-1}(0)$ and $\left[K: K^{\prime}\right]=p$.

Proposition 2.3. $D$ is nilpotent on $K$ if and only if $D^{p-1} \neq 0$ and $D^{p}=0$ on $K$.

Proof. Assume that $D$ is nilpotent on $K$. Since $\left[K: K^{\prime}\right]=p$, $D \neq 0$. Hence, $D^{p-1} \neq 0$ by Corollary 1.4 . Let $n$ be the minimum positive integer such that $D^{n}=0$ on $K$. By Proposition 1.8, there exists $a \in K^{\prime}$ such that $D^{p}=a D$. By the division algorithm, there exist nonnegative integers $q$ and $r<p$ such that $n=p q+r$. Then $0=D^{n}=\left(D^{p}\right)^{q} D^{r}=(a D)^{q} D^{r}=a^{q} D^{q} D^{r}=a^{q} D^{q+r}$ on $K$. Hence, either $a=0$, in which case we are done, or $D^{q+r}=0$, which implies $q+r \geq p q+r$, which is only possible if $q=0$, which further implies $D^{r}=0$, which contradicts the fact that $D^{p-1} \neq 0$.

The converse is obvious.

Corollary 2.4. Let $R$ be a subring of $K$ such that $D(R) \subset R$. Then $D$ is nilpotent on $K$ if and only if there exists $f \in R$ such that $D(f) \neq 0$ and $D^{2}(f)=0$.

Proof. If there exists $f \in R$ such that $D(f) \neq 0$ and $D^{2}(f)=0$, then by Lemma $1.10, D^{p}=0$. Conversely, if $D$ is nilpotent on $K$, then by Proposition 2.3, $D^{p}=0$ and there exists $g \in K$ such that $D^{p-1}(g) \neq 0$. After multiplying $g$ by an element of $R^{(p)}$, we may assume $g \in R$. If we let $f=D^{p-2}(g)$, then $f \in R, D(f) \neq 0$ and $D^{2}(f)=0$.

Remark 2.5. If $g \in k(x, y)$, it is easy to see that $D_{g}=0$ on $k(x, y)$ if and only if $g \in k\left(x^{p}, y^{p}\right)$. Also, if $g \notin k\left(x^{p}, y^{p}\right)$, then $k\left(x^{p}, y^{p}, g\right)=D_{g}^{-1}(0)$, and then clearly $\left[k(x, y): k\left(x^{p}, y^{p}, g\right)\right]=p$. 
Hence, if $g \notin k\left(x^{p}, y^{p}\right)$, then by Proposition 1.8, there exists a unique $\alpha_{g} \in D_{g}^{-1}(0)$ such that $D_{g}^{p}=\alpha_{g} D_{g}$.

Proposition 2.6. Assume $g \in k[x, y]$. Then $D_{g}$ is nilpotent if and only if $\nabla\left(g^{i}\right)=0$, for each $i=1,2, \ldots, p-1$, where $\nabla=$ $\partial^{2(p-1)} / \partial x^{p-1} \partial y^{p-1}$.

Proof. If $g \in k\left[x^{p}, y^{p}\right]$, then $D_{g}=0$ by Remark 2.5 and, clearly, $\nabla\left(g^{i}\right)=0$, for $i=1,2, \ldots, p-1$. So we may assume $g \notin k\left[x^{p}, y^{p}\right]$. Then, by Remark 2.5, we have $D_{g} \neq 0$, and there exists $\alpha_{g} \in$ $D_{g}^{-1}(0)$ such that $D_{g}^{p}=\alpha_{g} D_{g}$. By Proposition 1.5, $D_{g}^{p-1}(h)-\alpha_{g} h=$ $-\sum_{i=0}^{p-1} g^{p-1-i} \nabla\left(g^{i} h\right)$ for all $h \in k(x, y)$. Set $h=1$ to get $\alpha_{g}=$ $-\sum_{i=0}^{p-1} g^{p-1-i} \nabla\left(g^{i}\right)$. Since $\nabla(k[x, y])=k\left[x^{p}, y^{p}\right]$ and $g \notin k\left[x^{p}, y^{p}\right]$, $\alpha_{g}=0$ if and if $\nabla\left(g^{i}\right)=0$ for $i=1,2, \ldots, p-1$, but by Proposition 2.3, $D_{g}$ is nilpotent if and only if $\alpha_{g}=0$.

The next result shows that, when $D_{f}$ is nilpotent and $f^{+} \notin k\left[x^{p}, y^{p}\right]$, the conclusion of Lemma 2.1 holds for $f^{+}$. Since, in characteristic $p$, an $f$ that is part of a Jacobian pair is such that $D_{f}$ is nilpotent (Corollary 2.4), we might expect, in hopes of recovering Abhyankar's theorem, that if $D_{f}$ is nilpotent with $\operatorname{deg}(f)<p$, then $f$ has at most two points at infinity. Nevertheless, the examples in Proposition 2.8 show that Lemma 2.2 is not true when $\operatorname{deg}(f)<p$.

Corollary 2.7. Assume $f \in k[x, y]$ and $f^{+} \notin k\left[x^{p}, y^{p}\right]$. If $D_{f}$ is nilpotent, then there exists a homogeneous $H \in k[x, y]$ such that $D_{f^{+}}(H) \neq 0$ and $D_{f^{+}}^{2}(H)=0$.

Proof. We have $D_{f^{+}}$is nonzero and nilpotent on $k(x, y)$ by Remark 2.5, Proposition 2.6 and the fact that, for each positive integer $i, \nabla\left(f^{i}\right)=0$ implies $\nabla\left(\left(f^{+}\right)^{i}\right)=0$. By Corollary 2.4, there exists $h \in k[x, y]$ such that $D_{f^{+}}(h) \neq 0$ and $D_{f^{+}}^{2}(h)=0$. For each $i=0,1, \ldots, \operatorname{deg}(h)$, there exists homogenous $H_{i} \in k[x, y]$, with either $H_{i}=0$ or $\operatorname{deg}\left(H_{i}\right)=i$, such that $h=\sum_{i=0}^{\operatorname{deg}(h)} H_{i}$. Then

$$
D_{f^{+}}(h)=\sum_{i=0}^{\operatorname{deg}(h)} D_{f^{+}}\left(H_{i}\right),
$$


with each $D_{f^{+}}\left(H_{i}\right)$ equal to 0 or homogeneous of degree $i+\operatorname{deg}(f)-2$ and

$$
D_{f^{+}}^{2}(h)=\sum_{i=0}^{\operatorname{deg}(h)} D_{f^{+}}^{2}\left(H_{i}\right),
$$

with each $D_{f^{+}}^{2}\left(H_{i}\right)$ equal to 0 or homogeneous of degree $i+2 \operatorname{deg}(f)-4$. Since $D_{f^{+}}(h) \neq 0$, it follows that there exists a $j$ such that $D_{f+}\left(H_{j}\right) \neq$ 0 , and, since $D_{f^{+}}^{2}(h)=0$, it follows that $D_{f^{+}}^{2}\left(H_{j}\right)=0$.

Proposition 2.8. For the following homogeneous polynomials $G \in$ $k[x, y]$, we have that $G$ has $\operatorname{deg}(G)$ distinct linear factors in $k[x, y]$ and $D_{G}$ is nilpotent.

(i) any $G$ having distinct linear factors in $k[x, y]$ of degree that divides $p-2$

(ii) $G=x\left(x^{p-2}+y^{p-2}\right)$, for $p>3$;

(iii) $G=x\left(x^{(p-3) / 2}+y^{(p-3) / 2}\right)$, for $p>7$.

Proof. If $g \in k[x, y]$, Proposition 2.6 implies that $D_{g}$ is not nilpotent if and only if there exists some $i=1,2, \ldots, p-1$, such that $\nabla\left(g^{i}\right) \neq 0$. On the other hand, $\nabla\left(g^{i}\right) \neq 0$ if and only if $g^{i}$ has a monomial of the form $x^{r p-1} y^{s p-1}$ with nonzero coefficient for some positive integers $r$ and $s$. In the case where $G$ is homogeneous, the latter equivalence implies that, if $\nabla\left(G^{i}\right) \neq 0$, then $i \operatorname{deg}(G)=-2(\bmod p)$, and since $1 \leq i \leq p-1$, such an $i$ is unique. Therefore, if $G$ is homogeneous of degree less than $p, D_{G}$ is nilpotent if and only if $\nabla\left(G^{i}\right)=0$ for the unique $i=1,2, \ldots, p-1$, such that $i \operatorname{deg}(G)=-2(\bmod p)$.

In case (i), there exists a positive integer $m$ such that $m \cdot \operatorname{deg}(G)=$ $p-2$. Hence, the unique $i$ with $1 \leq i \leq p-1$ such that $i \operatorname{deg}(G)=$ $-2(\bmod p)$ is $i=m$. Since $G^{m}$ is homogeneous of degree $p-2, G^{m}$ has no monomials of the form $x^{r p-1} y^{s p-1}$ with nonzero coefficient, i.e., the degree of $G^{m}$ is too small. Hence, $\nabla\left(G^{m}\right)=0$.

In case (ii), with $G=x\left(x^{p-2}+y^{p-2}\right)$, the unique $i=1,2, \ldots, p-1$, such that $i \operatorname{deg}(G)=-2(\bmod p)$ is $i=2$. Then $G^{2}=x^{2 p-2}+2 x^{p} y^{p-2}+$ $x^{2} y^{2 p-4}$, and clearly $\nabla\left(G^{2}\right)=0$.

In case (iii), with $G=x\left(x^{(p-3) / 2}+y^{(p-3) / 2}\right)$, the unique $i=$ $1,2, \ldots, p-1$, such that $i \operatorname{deg}(G)=-2(\bmod p)$ is $i=4$. Then $G^{4}=$ $x^{2 p-2}+4 x^{(3 p-1) / 2} y^{(p-3) / 2}+6 x^{p+1} y^{p-3}+4 x^{(p+5) / 2} y^{(3 p-9) / 2}+x^{4} y^{2 p-6}$. 
Clearly, if $\nabla\left(G^{i}\right) \neq 0$, then $\nabla\left(x^{(p+5) / 2} y^{(3 p-9) / 2}\right) \neq 0$, which is the case only if $5 / 2=-1$ and $-9 / 2=-1(\bmod p)$, which is the case only if $p=7$.

From Corollary 2.7, we know that, for each of the homogeneous $G$ in Proposition 2.8, there exists homogeneous $H$ such that $D_{G}(H) \neq 0$ and $D_{G}^{2}(H)=0$, and yet each $G$ is of degree less than $p$ and has more than two distinct linear factors. However, there is no guarantee from Corollary 2.4 or Corollary 2.7 that $H$ will also have degree less than $p$. Thus, it is natural to ask if both $G$ and $H$ are homogeneous of degree less than $p$ such that $D_{G}(H) \neq 0$ and $D_{G}^{2}(H)=0$, then must $G$ have at most two distinct linear factors? The next example provides a negative answer in this case, also.

Proposition 2.9. Let $G=x y(x+y) \in k[x, y]$, where the characteristic of $k$ equals $p \neq 0$. If $p=2(\bmod 3)$, then $D_{G}$ is nonzero and nilpotent. In addition, there exists a homogeneous $H \in k[x, y]$ with $\operatorname{deg}(H)<p$ such that $D_{G}(H) \neq 0$ and $D_{G}^{2}(H)=0$.

Proof. Note that, if $1 \leq i \leq p-1$, then $\operatorname{deg}\left(G^{i}\right) \leq 3(p-1)$. Then, by the same argument used in the proof of Proposition 2.8, $\nabla\left(G^{i}\right) \neq 0$ for some $i=1,2, \ldots, p-1$, implies $3 i=2(p-1)$, which implies $p=1(\bmod 3)$. Hence, by Proposition $2.6, D_{G}$ is nilpotent. By Proposition $2.3, D_{G}^{p}=0$. Since $D_{G}(x)=G_{y} \neq 0$, there exists a positive integer $r \leq p-1$ such that $D_{G}^{r}(x) \neq 0$ and $D_{G}^{r+1}(x)=0$. Hence, if we let $H=D_{G}^{r-1}(x)$, then $D_{G}(H) \neq 0$ and $D_{G}^{2}(H)=0$. Also, by induction on $i$, we have for each $i \leq r, \operatorname{deg}\left(D_{G}^{i} x\right) \leq 1+i$. Hence, $\operatorname{deg}(H) \leq p-1$.

Conclusion 2.10. From Abhyankar, we know that in characteristic 0, a polynomial $f \in k[x, y]$ of positive degree has at most two points at infinity if there exists $g \in k[x, y]$ such that $J(f, g) \neq 0$ and $J\left(f^{+},(J(f, g))^{+}\right)=0$. Although this is not true in characteristic $p \neq 0$, the conclusion of Lemma 2.1 holds for polynomials of degree less than $p$ that are parts of Jacobian pairs by virtue of their defining nilpotent derivations. Since Lemma 2.2 also translates in characteristic $p$ to a statement about polynomials that define nilpotent derivations, it is natural to investigate the possible number of points at infinity of polynomials of degree less than $p$ that define nilpotent Jacobian 
derivations. However, as we have shown, such a polynomial can have as many points at infinity as its degree, thus proving, if indeed it is the case, that a polynomial of degree less than $p$ that is part of a Jacobian pair having at most two points at infinity will require a more holistic use of the Jacobian pair hypothesis. Yet, unless this can be proved, there does not seem to be a compelling reason to be optimistic about a reduction to characteristic $p$ approach to the Jacobian conjecture in characteristic 0 .

\section{REFERENCES}

1. Shareem S. Abhyankar, Expansion techniques in algebraic geometry, Tata Lect. Notes, 1977.

2. Jeffrey Lang, Purely inseparable extensions of unique factorization domains, Kyoto J. Math. 26 (1990), 453-471.

3. Jeffrey Lang and Satya Mandal, On Jacobian n-tuples in characteristic p, Rocky Mountain J. Math. 23 (1993), 271-279.

4. Pierre Samuel, Lectures on unique factorization domains, Tata Lect. Notes, 1964.

Mathematics Department, University of Kansas, Lawrence, KS, 66045

Email address: haras@ku.edu 